\title{
Application of thermography for microelectronic design and modelling
}

\author{
by G. De Mey ${ }^{1}$ and B. Więcek ${ }^{2}$
}

${ }^{1}$ University of Gent, ELIS, Sint Pieterniuwstraat 41, Gent, B-9000 Belgium, e-mail: demey@elis.rug.ac:be

${ }^{2}$ Technical University of Łódź, Institute of Electronics, Computer Thermography Group

18/22 Stefanowskiego St, 90-924 Łódź, Poland, e-mail: wiecek@ck-sg.p.lodz.pl

\begin{abstract}
This paper deals in with the particularities of infrared thermography in electronics or in other words with very small heat sources. This gives rise to very sharp temperature peaks and very rapid transient phenomena. For both of them, infrared thermography turns out to be a very effective measuring technique. At last some discussions about materials and combinations with modelling are presented. Fast thermal processes measurements in microelectronics are discussed using real-time image acquisition as well as so-called stroboscope method. Limits of using thermography for such investigations are outlined.
\end{abstract}

\section{Introduction}

In modern electronics and more specifically microelectronics the individual components are so good that the overall circuit performance (=frequency) is limited by other phenomena. First of all, one has to deal with the interconnection problem. The output signal generated by one transistor on a chip has to be transported to the input node of another subcircuit. To increase the working frequency, the distances of all these interconnections should be kept as short as possible. Hence components are made smaller to satisfy these requirements. However a problem arises. If components are placed closer together the heat generation per unit area increases too. The second problem is that the heat generation in a commonly used C-MOS circuit turns out to be proportional to the clock frequency. A Pentium processor at $150 \mathrm{MHz}$ clock frequency generates twice as much heat as the same processor operating at $75 \mathrm{MHz}$. Changing the clock frequency is used in portable computers to reduce the battery loading. The conclusion is obvious: processors have to run faster and faster so that the heat dissipation will continue to increase in the future. Even reducing the supply voltage will not stop this problem [1]. Silicon chip temperatures of 100 degrees or more are no longer exceptional any more.

It is quite evident to say that infrared thermography has a lot of applications in electronic design. One may wonder what is so typical about heat transfer in electronics or microelectronics. First of all, the dimensions of the individual heat sources are so small that the temperature distribution is showing very narrow peaks, which can only be seen through infrared thermography or liquid crystal thermography. Other techniques cannot be used at all. Secondly, the power dissipation and therefore the temperature distribution show very rapid transients. With infrared thermography it is possible to measure these time dependent phenomena quite accurately. In this paper some applications of thermography will be given. All of them are typical for the field of microelectronics.

\section{Trimmed resistors}

In telecommunication equipment, resistors are usually made with thick film technology. A conducting paste is screen printed through a screen on a ceramic substrate. The thickness of the layer cannot be changed by the designer. The only way to make different resistance values is to change the length and the width, or more generally the geometry. 
http://dx.doi.org/10.21611/qirt.1998.002

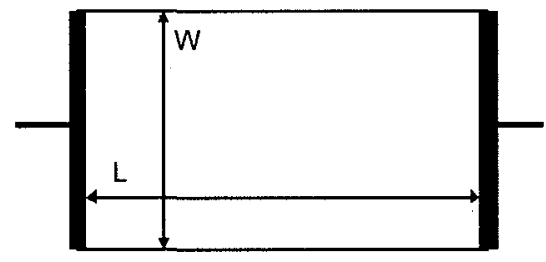

a

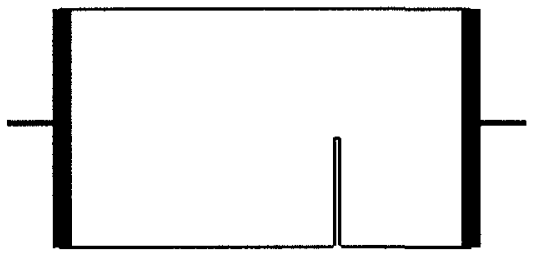

b

Fig. 1. A rectangular resistor as shown on fig.1a will have a resistance value given by: $R=R_{s q} \frac{L}{W}$ where $R_{s q}$ is the so-called square resistance, i.e. the resistance of a layout with a square geometry $(\mathrm{L}=\mathrm{W})$

If is often necessary to make resistors with a very high precision, which cannot be performed by only influencing $L$ or $W$. The resistive layers are then trimmed with a laser cutting a narrow channel and yielding a higher resistance value (Fig. 1b). Due to the trimming, the steam lines are no longer uniform. The power density is then no longer uniform either [2].



Fig. 2. Temperature distribution on the substrate for trimmed resistor

A hot spot will occur at the end of the trimming cut. Fig.2 shows a thermographic recording along a line going through the final point of the trim cut. This thermogram was made with an infrared microscope lens. One observes high peak temperatures up to 70 degrees. Only $100 \mu \mathrm{m}$ away from the peak the temperature already dropped to 40 degrees. This spatial behaviour explains why one will not burn his finger when making a thermal contact with the resistor. The thermal receptors in the fingertips are somewhat at a depth of 300 microns, so that one will never sense the maximum temperature.

\section{Fast thermal transients}

Voltages and currents in microelectronic components vary very rapidly. As a consequence the power dissipation is also a time dependent function involving very short time constants. There is a general believe that these thermal transients are not very 
important at all. Only the mean power dissipation has to be taken into account in order to determine the time averaged temperature distribution, and this is sufficient for a complete thermal design. However, this statement is far from being true. In order to understand this contradiction, consider a cross section of a typical integrated circuit package. On the silicon surface, one has a large amount of heat sources, each of them having dimensions of $1 \mu \mathrm{m}$ or even less. They all produce heat pulses at frequencies of $100 \mathrm{MHz}$ or higher. It is quite straightforward that these rapid small-scale temperature variations will not extend to the exterior of the package. A normal thermographic inspection of packed circuits will never reveal these particular phenomena. In order to observe these, one has to look directly on the silicon's surface. These kinds of studies are very important because these temperature fluctuations give rise to thermal feedback. All semiconductor characteristics are temperature dependent. A sudden temperature change due to self-heating or due to any heat source in the vicinity will influence the electric behaviour of the circuit. Unwanted signals can be induced due to this (usually unforeseen) thermal coupling.

With a normal thermographic camera it is possible to investigate fast thermal phenomena. Most thermographic cameras use a spatial scanning system, which means that each pixel on the screen corresponds to a temperature measurement done in a very short time. By using the stroboscopic principle i.e. the electric input signal is synchronised with the thermographic scanner. The temperature can then be readout with a much higher time resolution. With focal plane array cameras this technique is not possible.

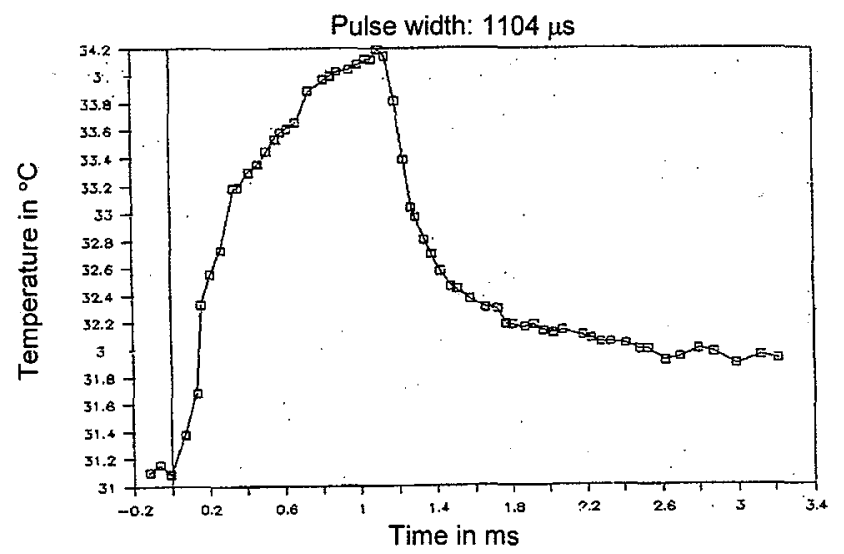

Fig. 3. Temperature evolution during pulse power excitation

Using the stroboscopic principle, a rectangular non-trimmed thick film resistor was heated periodically [3]. The resistor being very thin, one can treat the problem as a surface heat source placed on a ceramic substrate. If a constant power pulse $\left(p\left[\mathrm{~W} / \mathrm{m}^{2}\right]\right)$ is applied, the temperature varies according to:

$$
T=\frac{2 p \sqrt{t}}{\sqrt{\pi k C_{v}}}
$$

where: $k$ - thermal conductivity of the substrate, $C_{v}$ - its thermal capacity per unit volume and $t$ is the time.

Fig. 3 shows the experimental results. Temperature increases of $1^{\circ} \mathrm{C}$ in $30 \mu \mathrm{s}$ have been recorded experimentally. A very good agreement with the $\sqrt{t}$ behaviour was found too. 
http://dx.doi.org/10.21611/qirt.1998.002

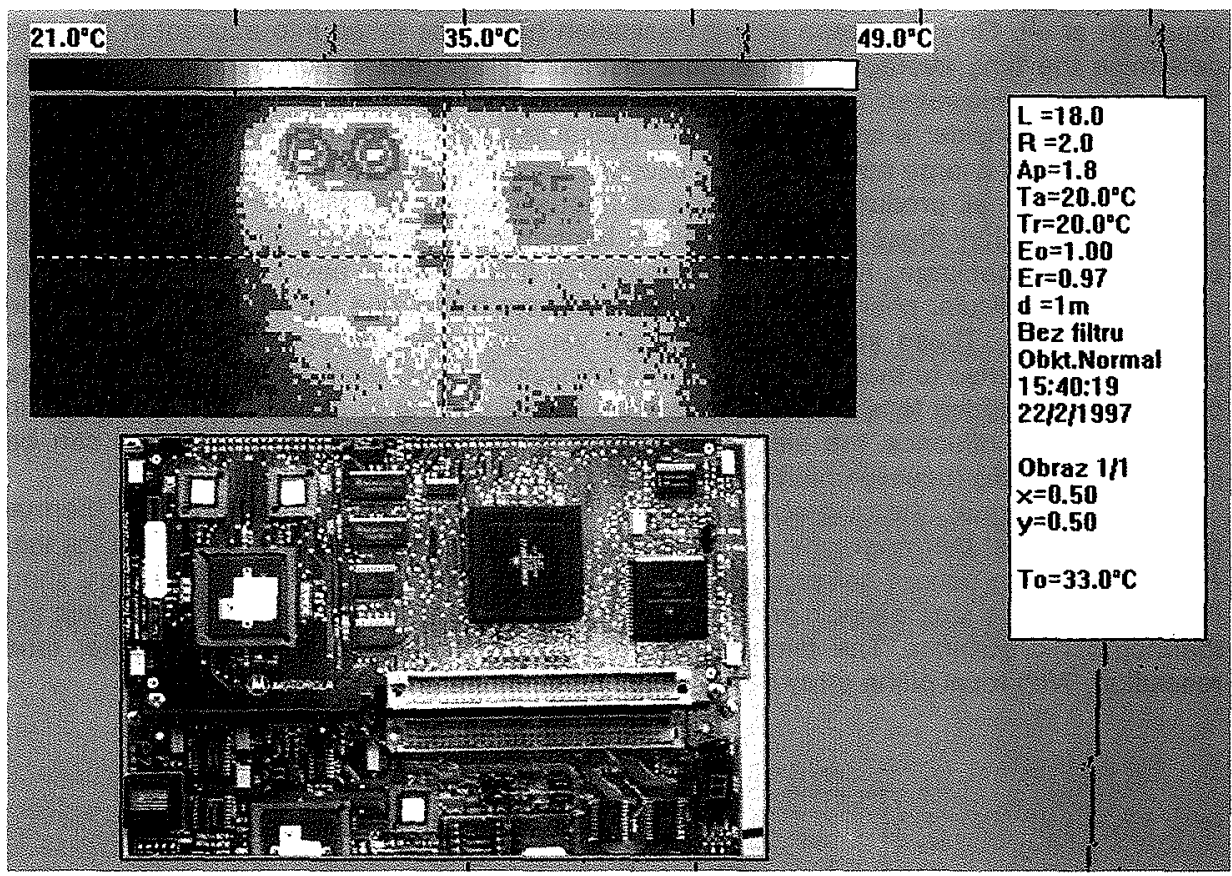

Fig. 4. Thermal and visual images of electronic circuit with hot spots

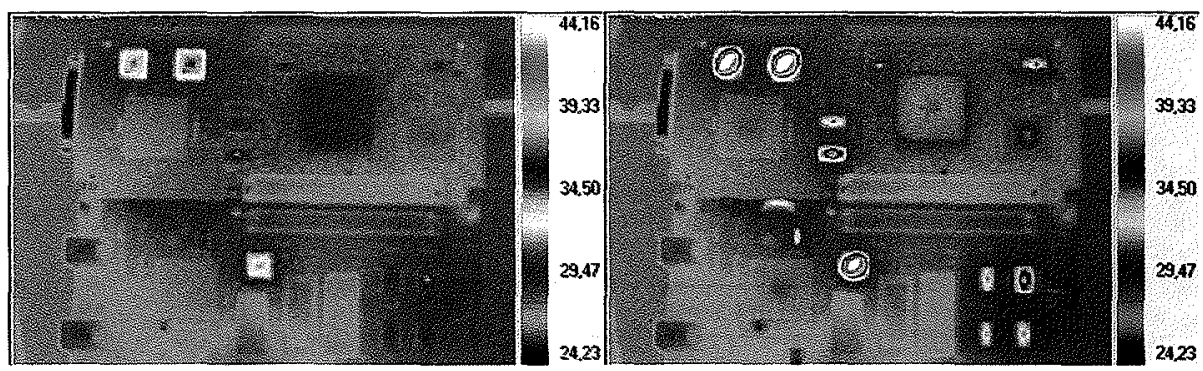

Fig. 5. Dynamic thermal process

\section{Influence of the camera}

If a thermographic camera is focussed on a screen at a given temperature TS, all radiation emitted from the point $P$ within a solid angle $\alpha$ is falling on the lens and deflected to the detector, usually at a very low temperature $T D$, which can be as low a $-200^{\circ} \mathrm{C}$.

Depending on the scanning system, this situation occurs not permanently for the same point $P$. For focal plane array cameras, this condition is more or less permanent. In this way a thermographic camera can influence the temperature of the device under test. However, the lens aperture being much smaller than the distance to the object, this effect is negligible.

The situation becomes different if infrared microscopy is used, which is a quite common job in microelectronics. The first lens of a microscope objective is quite close to the object, and the solid angle $\alpha$ becomes then relatively big. The camera will then influence the temperature of the microcircuit. A correction of the measured temperature is then necessary. 


\section{Materials}

In microelectronics a lot of materials are used like semiconductors ( $\mathrm{Si}, \mathrm{GaAs}$, $\ln \mathrm{P}, \ldots)$, metals $(\mathrm{Al}, \mathrm{Au}, \mathrm{Ag}, \ldots)$, and ceramics $\left(\mathrm{Al} 2 \mathrm{O} 3, \mathrm{AlN}, \mathrm{BeO}, \mathrm{C}_{1} \ldots\right.$ ). All these materials just mentioned are far from being good infrared emitters. Semiconductors are transparent, metals reflect the IR radiation, and ceramics have poor emissivities. The only way to use thermography is to paint the circuit with a good emitting coating.

One will argue that such a coating will influence the thermal properties of the circuits. In many cases the coating is much thicker than the original heat source. The best way to overcome these problems is to simulate the thermal behaviour taking the coating into account and compare the results with thermographic recordings. A subsequent simulation without any coating will then provide realistic temperature values.

\section{Comparison with modelling}

An obvious application of infrared thermography is the comparison between experimental and simulation results. This is a must in order to test the algorithms used for the simulation tools. In electronics such comparisons are compulsory because the thermal properties like conductivity are only known for bulk materials. Quite different values can be obtained if the same materials are made in small sized devices.

As an example we can mention the fact that alumina $(96 \%)$ has a thermal conductivity of about $k=20 \mathrm{~W} /(\mathrm{m} \cdot \mathrm{K})$. Measurements done on hybrid circuits made on alumina substrates (thickness $0.6 \mathrm{~mm}$ ) gave rise to values up to $k=27 \mathrm{~W} /(\mathrm{m} \cdot \mathrm{K})$.

\section{In situ testing of electronic circuits}

A very interesting application of thermography is the testing of circuits during production. By applying power to a circuit a temperature distribution will be set up as a function of time. There is no need to wait till steady conditions are reached. If one records a thermogram say after 10 seconds, this thermogram can be compared with a stored thermogram of a known good circuit. If deviations are too large the circuit can be removed from the production. One can only be surprised that this technique is not commonly used in industry.

\section{Conclusion}

In this paper some special features of thermography in the world of electronics and microelectronics have been explained. Especially the very small dimensions of the heat sources make it very attractive for thermographic investigations.

\section{REFERENCES}

[1] SZEKELY (V.), RENCZ (M.), TOROK (S.), FARKAS (F.) and MARTA (Cs.) - Advances in the thermal testing of IC's. 3rd Therminic workshop, 1997, Cannes, p. 5-10.

[2] CASSELMAN (G.) and DE MEY (G.) - Influence of trimming on the electric field distribution in thick film resistors. Hybrid Circuits, 1985, vol.8, p.10-12.

[3] DE MEY (G.) - Thermographic measurements of hybrid circuits. 7th European Hybrid Microelectronics Conference, Hamburg, 1989, proceedings p.1-7.

[4] DE MEY (G.), BOONE (E.), DE SMET (W.), COLPAET (G.), NACHTERGAELE (G.) and DEMOLDER (S.) - Measuring thermal properties in thin multilayer structures. 8th European Hybrid Microelectronics Conference, Rotterdam, 1991, proceedings p. 360-367.

[5] WIĘCEK (B.), DE MEY (G.) - Evaluation of heat dissipation by natural convection for VLSI circuits, Proc. TERMINIC'96 Conf., Budapest, Sept.25-27, 1996.

[6] WIEcCEK (B.), DE BEATSELIER (E.), DE MEY (G.) - Active Thermography Application for Solder Thickness Measurement, Proc. TERMINIC'96 Conf., Budapest, Sept.25-27, 1996. 